\title{
Rapid and complete radiological resolution of an intradural cervical cord lung cancer metastasis treated with spinal stereotactic radiosurgery: case report
}

\author{
E. Emily Bennett, MD, ${ }^{1,2}$ Camille Berriochoa, MD, ${ }^{3,4}$ Ghaith Habboub, MD, ${ }^{1,2}$ Scott Brigeman, MD, ${ }^{5}$ \\ Samuel T. Chao, MD, ,4,6 and Lilyana Angelov, MD, FRCS(C) $)^{1,2,4,6}$

\begin{abstract}
${ }^{1}$ Department of Neurological Surgery, ${ }^{2}$ Neurological Institute, ${ }^{3}$ Department of Radiation Oncology, ${ }^{4}$ Taussig Cancer Institute, and ${ }^{6}$ The Rose Ella Burkhardt Brain Tumor and Neuro-Oncology Center, Cleveland Clinic; and ${ }^{5}$ Case Western Reserve University Medical School, Cleveland, Ohio
\end{abstract}

\begin{abstract}
Stereotactic radiosurgery (SRS) has emerged as a treatment option for patients with spinal metastatic disease. Although SRS has been shown to be successful in a multitude of extradural metastatic tumors causing cord compression, very few cases of intradural treatment have been reported. The authors present a rare case of an intradural extramedullary metastatic small cell lung cancer lesion to the cervical spine resulting in cord compression in an area that had also been extensively pretreated with conventional external-beam radiation therapy. The patient underwent successful SRS to this metastatic site, with rapid and complete resolution of his lesion.
\end{abstract}

https://thejns.org/doi/abs/10.3171/2016.9.FOCUS16254

KEY WORDS spinal metastasis; spinal cord compression; small cell lung carcinoma; spinal stereotactic radiosurgery

$\mathrm{S}$ PINAL metastases are an unfortunate sequela in up to $80 \%$ of all patients with solid tumors, and most frequently present with back pain. ${ }^{8}$ When left untreated, metastatic disease can also progress to cord compression, causing permanent loss of neurological function. ${ }^{11}$ The majority of metastatic lesions are located extradurally, with only $5 \%$ of tumors presenting intradurally. ${ }^{4,9}$ However, despite the presence of spinal metastases, some patients may have prolonged survival; treatment is therefore indicated to decrease the tumor burden, alleviate pain, prevent or minimize neurological decline, and improve overall quality of life. ${ }^{11}$

Spinal metastasis treatments can comprise stand-alone monotherapies including conventional external-beam radiation therapy (cEBRT), spinal stereotactic radiosurgery (SRS), surgery, and occasionally chemotherapy. However, treatment often requires multimodality regimens. Many factors are important in the overall decision-making process in the treatment of patients with metastatic spine disease. These factors include the neurological status of the patient, location of the tumor and degree of cord compression, oncological factors such as primary tumor histological type and expected response to radiation, the burden of disease and available treatment options, the presence of mechanical instability, and the overall health status of the patient, including other comorbid diseases. ${ }^{3,6,11}$

Stereotactic radiosurgery is a more recent advancement that offers many advantages over cEBRT in treating metastatic spinal lesions. This focal therapy can be safely administered in 1-5 sessions-allowing delivery of a tumoricidal, large dose of radiation into the tumor-while its precision in targeting and rapid dose falloff spares the spinal cord and other organs at risk from radiation. ${ }^{13} \mathrm{In}$ contrast to SRS, cEBRT requires multisession treatment, which can delay essential chemotherapy. Furthermore, the palliative cEBRT dose is generally limited due to radiation intolerance of the adjacent spinal cord, and can often be insufficient for effective or durable control in the setting of radioresistant tumors.

Management of spine tumors is markedly more chal-

ABBREVIATIONS BPI = Brief Pain Inventory; cEBRT = conventional external-beam radiation therapy; IMRT = intensity-modulated radiation therapy; $\mathrm{RT}=$ radiation therapy; SCLC = small cell lung cancer; SRS = stereotactic radiosurgery; WBRT = whole-brain RT.

SUBMITTED June 17, 2016. ACCEPTED September 21, 2016.

INCLUDE WHEN CITING DOI: 10.3171/2016.9.FOCUS16254. 
lenging in patients who have already been treated with cEBRT; in these patients additional conventional radiation therapy (RT) at recurrence can potentially increase the risk of spinal cord toxicity or radiation myelopathy. ${ }^{12}$ In contrast, Sahgal et al. found that if SRS treatment is > 5 months after cEBRT, the risk of radiation myelopathy is low if the total normalized biologically effective dose does not exceed $70 \mathrm{~Gy},{ }^{12}$ and SRS has been successfully used to safely treat metastatic tumors in patients with previous localized cEBRT.,12 However, although SRS has been shown to be successful in treating a multitude of extradural metastatic tumors causing cord compression, few cases have been reported in which reirradiation was performed using SRS in treating intradural metastatic disease that occurred following cEBRT. ${ }^{12,14}$

We present a rare case of an intradural extramedullary metastatic small cell lung cancer (SCLC) lesion to the cervical spine resulting in cord compression in an area of the cervical cord that had been extensively pretreated with cEBRT for definitive management of oral cavity carcinoma that had been initially diagnosed 2 years prior, followed by a diagnosis of limited-stage SCLC 6 months prior. The patient underwent successful SRS to this metastatic site, with rapid and complete resolution of his lesion and no immediate toxicity related to treatment.

\section{Case Report}

History and Examination

A 53-year-old right-handed man with a complicated oncological history was referred from another hospital to our clinic with known metastatic SCLC and a new ventral C1-2 intradural extramedullary cervical lesion. The patient had a history of Stage III right retromolar trigone oral cavity cancer for which he underwent resection followed by adjuvant cEBRT; treatment was completed in February 2005 . At that time he received a total dose of 70.2 Gy in 39 fractions, with 39.6 Gy in 22 fractions delivered to the upper cervical cord. Several months later he was found to have a new left lower lobe pulmonary lesion and underwent a partial lobectomy. Pathological investigation confirmed SCLC, with further systemic staging demonstrating Stage IIIB limited-stage SCLC. He received adjuvant cEBRT totaling 64.8 Gy to this site. Shortly thereafter he was found to have metastatic intracranial disease, for which he underwent resection followed by whole-brain radiation therapy (WBRT) - 40 Gy in 20 fractions, with an additional posterior fossa boost to $10 \mathrm{~Gy}$ in 5 fractions. The WBRT was completed in August 2005.

Later that year, he developed severe neck pain without other neurological symptoms. His pain was not well controlled despite pain medications as well as long-term steroids. The MRI studies of his cervical spine revealed a ventral contrast-enhancing intradural extramedullary mass centered at C1-2 that was causing displacement of the cervical cord, at which point he presented to our institution (Fig. 1). The patient was referred to our institution for management of de novo cervical spine pain, and this mass was identified on a dedicated cervical MRI study specifically obtained for the evaluation of this new neck pain. No prior cervical imaging studies were available

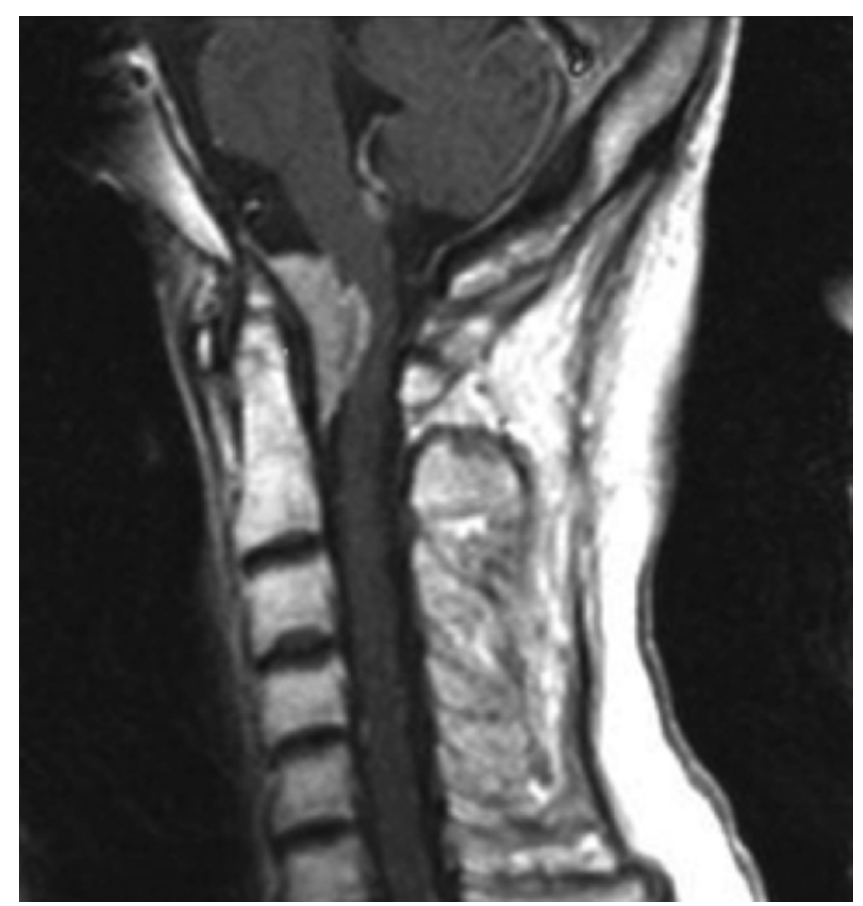

FIG. 1. Sagittal MR image of the cervical spine obtained with gadolinium contrast in the $\mathrm{T} 1$ phase before spinal radiosurgery.

for comparison; however, in the context of his previous cancer, the cervicomedullary junction would have been at least partially imaged and thus any lesions would have been identified (if present) prior to his presentation at our institution. The patient's pain appeared to be directly related to his intradural tumor because no degenerative pathology was observed that could explain his pain. He also had partial pain relief with opioid analgesics and decadron, further supporting a tumor-related etiology of his neck pain. At initial evaluation, his neurological examination showed no gross motor or sensory deficits and was notable only for myelopathy presenting with hyperreflexia in his upper and lower extremities. His Karnofsky Performance Scale score was 70 .

His case was discussed at our institution's tumor board, which is composed of a multidisciplinary team consisting of neurosurgeons, radiation oncologists, medical oncologists, neuro-oncologists, and radiologists. The patient was believed to be a poor surgical candidate given his systemic disease and suboptimal nutritional status. However, given the size of the mass and the likely progression to quadriparesis and possibly respiratory compromise if left untreated, it was recommended that he undergo some form of palliative radiation treatment for management of the cervical mass. The risks and benefits of cEBRT versus SRS were discussed. The tumor board's final recommendations were for the more targeted treatment option of SRS, given the history of multiple courses of cEBRT previously delivered to the area and concerns regarding treatment-related myelopathy unless a specifically cordsparing radiation treatment modality was performed. The patient was counseled on the increased risk associated with radiation to his lesion given the location and history of prior cEBRT to the region, and he agreed to proceed. 

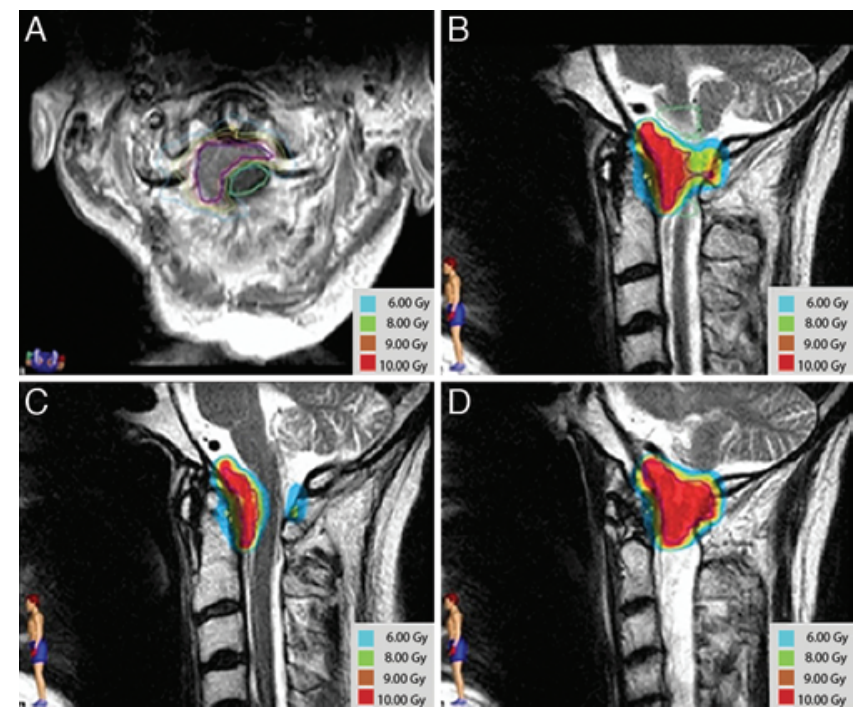

FIG. 2. Axial (A) and sagittal (B-D) views of the reconstructed CT/MRI fusion images. These images show a prescribed dose of $10 \mathrm{~Gy}$ in $1 \mathrm{frac}-$ tion via 7 IMRT fields to the $100 \%$ isodose line, using 6-MV photons.

\section{Treatment Course}

Per our protocol, the patient underwent CT-based simulation in the supine position while immobilized in a 5-point head and neck mask. These images were then fused to his high-definition MR image (1.5-mm slice thickness, 1-mm gap) using iPlan (Brainlab AG). After image acquisition, the MR and CT images were precisely coregistered, and tumor and the organs at risk, including the spinal cord, were identified and contoured. At the Cleveland Clinic, we define the spinal cord as an organ at risk with no margin, and the cord volume is contoured at the vertebral level(s) of interest and $3 \mathrm{~mm}$ cranial and caudal to the tumor region. Furthermore, we limit $\leq 10 \%$ of the contoured spinal cord to $\geq 10 \mathrm{~Gy}$, and we limit the maximum point dose within the cord to $14 \mathrm{~Gy} .^{2} \mathrm{~A}$ treatment plan was then devised and carefully reviewed based on these principles (Fig. 2).

On the day of treatment, the patient was repositioned and immobilized in the 5-point head and neck mask. Accurate repositioning was achieved and radiographically confirmed with films and ExacTrac (Brainlab). The lesion was treated to a prescribed dose of 10 Gy in 1 fraction via 7 intensity-modulated radiation therapy (IMRT) fields to the $100 \%$ isodose line, using 6-MV photons. The fields covered $86.8 \%$ of the target. A more conservative dose of 10 Gy was deliberately used, given the amount of prior radiation to which the spinal cord had been exposed during the previous treatment of adjacent disease. A total target volume of $5.26 \mathrm{~cm}^{3}$ was treated with a maximum dose of 11.2 Gy and average conformality index of 1.52 .

\section{Postoperative Course}

Following spinal SRS, the patient had almost complete resolution of his neck pain as confirmed by his 4 contacts (3 telephone conversations and 1 office visit), which took place post-SRS (Table 1). Furthermore, we use the Brief Pain Inventory (BPI) data prospectively collected in our patients to obtain estimates of their pain prevalence and severity. As measured by his BPI, his pain scores de-
TABLE 1. The BPI scores in a patient with metastasis to the spine treated with SRS

\begin{tabular}{|c|c|c|}
\hline $\begin{array}{c}\text { BPI } \\
\text { Pain Metric }\end{array}$ & $\begin{array}{l}\text { Pre- } \\
\text { SRS }\end{array}$ & $\begin{array}{l}\text { Post- } \\
\text { SRS }\end{array}$ \\
\hline \multicolumn{3}{|l|}{ During the past 3 days, pain score: } \\
\hline At its worst & $7 / 10$ & $2 / 10$ \\
\hline On average & $5 / 10$ & $3 / 10$ \\
\hline Right now & $4 / 10$ & $2 / 10$ \\
\hline \multicolumn{3}{|l|}{ During the past 3 days, pain has interfered $w /$ your: } \\
\hline General activity & $5 / 10$ & $4 / 10$ \\
\hline Mood & $5 / 10$ & $2 / 10$ \\
\hline Walking ability & $5 / 10$ & $2 / 10$ \\
\hline Normal work & $10 / 10$ & $2 / 10$ \\
\hline Relations w/ other people & $8 / 10$ & $2 / 10$ \\
\hline Sleeping & $10 / 10$ & $2 / 10$ \\
\hline Enjoyment of life & $9 / 10$ & $2 / 10$ \\
\hline Morphine equivalents & 6.8 & 2.3 \\
\hline $\begin{array}{l}\text { In the last } 24 \text { hrs, how much relief have pain treat- } \\
\text { ments or medications provided? }\end{array}$ & $25 \%$ & $90 \%$ \\
\hline
\end{tabular}

BPI scale: 0-10 (No pain = $012345678910=$ Pain as bad as you can imagine). Scale for pain interference: $0-10$ (Does not interfere $=01234567$ 8910 = Complete interference).

creased by $67 \%$ compared with before SRS. His narcotics use, as measured by morphine equivalents, also decreased by $66 \%$. Neurologically he remained without deficit except for ongoing mild tingling in his fingers that was thought to be related to his systemic therapy. This pain improvement was associated with complete resolution of his lesion on his MRI study obtained at 3 weeks posttreatment (Fig. 3). Despite our frequent postintervention contact, our followup duration is limited because the patient died 4 weeks after treatment due to progression of systemic disease.

\section{Discussion}

We present a case of SCLC with intradural metastasis to the spine resulting in cord compression that completely resolved on MRI within weeks of SRS. The treatment was associated with significant improvement in pain and quality of life.

There are few case reports of successful SRS for metastatic intradural lesions. Shin et al. reported on 9 patients with intradural metastatic lesions, 4 of which were intradural and extramedullary. Two patients had been previously treated with cEBRT, but the details of their prior treatments were not reported. These investigators found that SRS with a mean treatment dose of $13.8 \mathrm{~Gy}$ in a single session improved presenting symptoms in $80 \%$ of patients. ${ }^{14}$ Furthermore, only 1 patient in that series went on to have further progression of their disease. No radiation toxicity was seen during the average 10-month follow-up.

Additionally, Mori et al. evaluated SRS in 3 patients with intradural metastases who also had undergone previous cEBRT. ${ }^{7}$ These authors used 24-36 Gy in 5-12 fractions to treat the lesions. They found that all tumors were stable or decreased in size, with follow-up durations rang- 


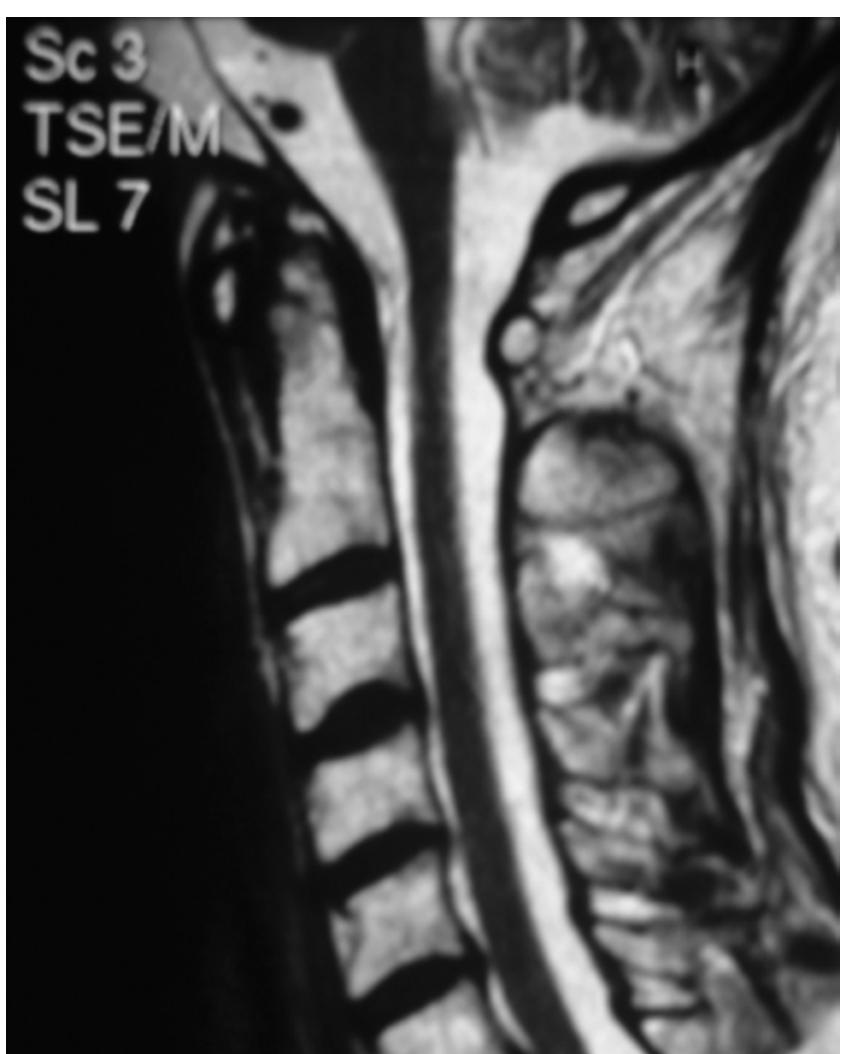

FIG. 3. Sagittal MRI sequence of the cervical spine in the T2 phase obtained without gadolinium after spinal radiosurgery.

ing from 5 to 22 months. Neurological symptoms were also stable or improved in all of their patients. Although those authors added unique cases of radiation treatment for intradural spinal metastatic lesions following previous cEBRT, the radiation used in their series is highly conformal fractionated radiotherapy, which is distinct from true SRS. Similar to Shin et al., we treated with a maximum dose of $11.2 \mathrm{~Gy}$ in a single fraction; however, the patient discussed in this case report was heavily treated with prior cEBRT, thus making this a unique contribution to the literature.

Radiation-induced myelopathy is the most serious complication following SRS, and usually presents between 6 months and 3 years after treatment. ${ }^{1}$ There are limited data about spinal cord tolerance of a single-dose radiosurgery for intradural lesions, and most decisions regarding treatment dosing are made using surrogate recommendations. Ryu et al. concluded that at least $10 \mathrm{~Gy}$ to $10 \%$ of spinal cord volume (defined as $6 \mathrm{~mm}$ above and below the radiosurgery target) appears to be within cord tolerance for single-fraction radiosurgical treatment..$^{10}$ Furthermore, Sahgal et al. evaluated 5 patients with and 19 patients without radiation myelopathy following SRS and concluded that $10 \mathrm{~Gy}$ to a maximum point of the spinal cord is not associated with significant cord toxicity. ${ }^{12}$ Kirkpatrick et al. specifically evaluated partial cord irradiation and they recommended a maximum cord dose of $13 \mathrm{~Gy}$ in a single fraction. ${ }^{5}$ These authors found that 13 Gy dose to be associated with a $<1 \%$ risk of injury to the spinal cord. Our case adds our findings to those of Shin et al., that in treat- ing intradural lesions, limiting $\leq 10 \%$ of the contoured spinal cord to $\geq 10 \mathrm{~Gy}$ in a single fraction is safe and effective, at least in the short term. ${ }^{14}$

Our patient had undergone multiple cEBRT treatments in the local area that precluded him from receiving further cEBRT. Treatment with SRS has become an acceptable alternative in cases of spinal compression and reirradiation. As described above, and as reported by Mori et al., 3 patients who had intradural lesions were also successfully treated with reirradiation using SRS after previous cEBRT. Our patient received 36.9 Gy to his cervical spinal cord during treatment for his Stage III right retromolar trigone oral cavity cancer during an era of 3D conformal radiotherapy for head and neck cancer. He later received $50 \mathrm{~Gy}$ at $2 \mathrm{~Gy} /$ fraction to his posterior fossa (and adjacent cervical cord) as a part of his WBRT. Thus, a total dose exceeding $80 \mathrm{~Gy}$ in cEBRT was delivered to this site prior to his SRS; hence, we used a more modest dose of 10 Gy to this area in a single session. It is important to note that in this clinical setting, our patient did not develop any signs of acute cord toxicity following treatment, despite his prior radiotherapy. However, we acknowledge that our follow-up was relatively short because of the patient's systemic progression and that the effects of spinal cord toxicity may not have had time to fully manifest.

Although we did not obtain a biopsy of his intradural tumor prior to treatment, his lesion was most consistent with an SCLC, given the intradural presentation and the rapid resolution of the lesion within 3 weeks of SRS. Furthermore, his symptoms had almost completely resolved following treatment. Given his history of multifraction radiotherapy, this patient was precluded from receiving additional cEBRT to this newly discovered lesion, because the upper cervical spinal cord would be subject to an unacceptable amount of radiation. Also, surgical decompression was not a viable option, given the ventral upper cervical location of the mass combined with the patient's poor overall medical condition.

Stereotactic radiosurgery allowed for a single palliative outpatient treatment that was minimally invasive, rapidly effective in terms of both pain and local tumor control, well tolerated, and convenient for the patient. One may argue, given the short survival in this particular patient following treatment, that therapy should have been limited to medical management with analgesics only. At the time of his treatment we could not predict his short course, and improving his quality of life was our primary goal, which was achieved rapidly following treatment. Given the preservation of neurological function as well as the extent and speed of pain relief experienced by many patients treated with SRS (at times obviating the need for high-dose opiate analgesics and the associated debilitating cognitive impairment that often ensues), this therapeutic strategy is now considered by many to be an important and valuable tool in the overall management of patients during the terminal stages of their cancer. Thus, SRS is an important technological advancement that often results in a global improvement of a patient's quality of life, and it offers important therapeutic options in patients (especially in the palliative setting) for whom typically few treatment options exist. 


\section{References}

1. Abbatucci JS, Delozier T, Quint R, Roussel A, Brune D: Radiation myelopathy of the cervical spinal cord: time, dose and volume factors. Int J Radiat Oncol Biol Phys 4:239248, 1978

2. Balagamwala EH, Angelov L, Koyfman SA, Suh JH, Reddy CA, Djemil T, et al: Single-fraction stereotactic body radiotherapy for spinal metastases from renal cell carcinoma. J Neurosurg Spine 17:556-564, 2012

3. Bilsky MH, Laufer I, Burch S: Shifting paradigms in the treatment of metastatic spine disease. Spine (Phila Pa 1976) 34 (22 Suppl):S101-S107, 2009

4. Joaquim AF, Ghizoni E, Tedeschi H, Pereira EB, Giacomini LA: Stereotactic radiosurgery for spinal metastases: a literature review. Einstein (Sao Paulo) 11:247-255, 2013

5. Kirkpatrick JP, van der Kogel AJ, Schultheiss TE: Radiation dose-volume effects in the spinal cord. Int J Radiat Oncol Biol Phys 76 (3 Suppl):S42-S49, 2010

6. Laufer I, Rubin DG, Lis E, Cox BW, Stubblefield MD, Yamada Y, et al: The NOMS framework: approach to the treatment of spinal metastatic tumors. Oncologist 18:744-751, 2013

7. Mori Y, Hashizume C, Shibamoto Y, Kobayashi T, Nakazawa H, Hagiwara M, et al: Stereotactic radiotherapy for spinal intradural metastases developing within or adjacent to the previous irradiation field-report of three cases. Nagoya J Med Sci 75:263-271, 2013

8. Nielsen OS: Palliative radiotherapy of bone metastases: there is now evidence for the use of single fractions. Radiother Oncol 52:95-96, 1999

9. Perrin RG, Livingston KE, Aarabi B: Intradural extramedullary spinal metastasis. A report of 10 cases. J Neurosurg 56:835-837, 1982

10. Ryu S, Jin JY, Jin R, Rock J, Ajlouni M, Movsas B, et al: Partial volume tolerance of the spinal cord and complications of single-dose radiosurgery. Cancer 109:628-636, 2007

11. Ryu S, Yoon H, Stessin A, Gutman F, Rosiello A, Davis R: Contemporary treatment with radiosurgery for spine metastasis and spinal cord compression in 2015. Radiat Oncol J 33:1-11, 2015
12. Sahgal A, Ma L, Weinberg V, Gibbs IC, Chao S, Chang UK, et al: Reirradiation human spinal cord tolerance for stereotactic body radiotherapy. Int J Radiat Oncol Biol Phys 82:107-116, 2012

13. Seung SK, Larson DA, Galvin JM, Mehta MP, Potters L, Schultz CJ, et al: American College of Radiology (ACR) and American Society for Radiation Oncology (ASTRO) Practice Guideline for the Performance of Stereotactic Radiosurgery (SRS). Am J Clin Oncol 36:310-315, 2013

14. Shin DA, Huh R, Chung SS, Rock J, Ryu S: Stereotactic spine radiosurgery for intradural and intramedullary metastasis. Neurosurg Focus 27(6):E10, 2009

\section{Disclosures}

The authors report no conflict of interest concerning the materials or methods used in this study or the findings specified in this paper.

\section{Author Contributions}

Conception and design: Angelov. Acquisition of data: Bennett, Habboub, Brigeman. Analysis and interpretation of data: Bennett, Angelov. Drafting the article: Bennett, Habboub, Brigeman. Critically revising the article: Angelov, Bennett, Berriochoa, Chao. Reviewed submitted version of manuscript: Angelov, Bennett, Berriochoa, Chao. Approved the final version of the manuscript on behalf of all authors: Angelov. Study supervision: Angelov.

\section{Supplemental Information \\ Previous Presentations}

This paper has not been previously published. However, our work was accepted as an abstract for electronic poster to the Congress of Neurological Surgeons 2016 Annual Meeting.

\section{Correspondence}

Lilyana Angelov, Department of Neurological Surgery, Cleveland Clinic Foundation, 9500 Euclid Ave., Cleveland, OH 44195. email: angelol@ccf.org. 